\title{
Variational approach to dequantization
}

\author{
Ricardo A. Mosna* \\ Departamento de Matemática, Universidade Estadual de Campinas, CP 6065, 13083-859, Campinas, Brazil. \\ I. P. Hamilton $\dagger$ \\ Department of Chemistry, Wilfrid Laurier University, Waterloo, Canada N2l 3C5. \\ L. Delle Sit€ $¥$ \\ Max-Planck-Institute for Polymer Research, Ackermannweg 10, D 55021, Mainz, Germany.
}

(Dated: September 22, 2018)

\begin{abstract}
We present a dequantization procedure based on a variational approach whereby quantum fluctuations latent in the quantum momentum are suppressed. This is done by adding generic local deformations to the quantum momentum operator which give rise to a deformed kinetic term quantifying the amount of "fuzzyness" caused by such fluctuations. Considered as a functional of such deformations, the deformed kinetic term is shown to possess a unique minimum which is seen to be the classical kinetic energy. Furthermore, we show that extremization of the associated deformed action functional introduces an essential nonlinearity to the resulting field equations which are seen to be the classical Hamilton-Jacobi and continuity equations. Thus, a variational procedure determines the particular deformation that has the effect of suppressing the quantum fluctuations, resulting in dequantization of the system.
\end{abstract}

PACS numbers: 03.65.Ta, 45.10.Db, 11.10.Ef

Quantum mechanics is an extremely successful theory for the description of atomic and molecular systems. Its predictions of microscopic phenomena are highly accurate and it is unrivalled as a physical theory. On the other hand, because of the undisputable success of classical mechanics in its domain of validity, there is continued interest in dequantization procedures whereby the classical regime is obtained from the quantum one. Here by dequantization we do not mean the procedure of obtaining a semiclassical limit of a given quantum system, as in the WKB approximation. Rather, following [1], by dequantization we mean "a set of rules which turn quantum mechanics into classical mechanics".

An insightful step towards dequantization is the introduction of formulations of classical mechanics that are operator based. The earliest such formulation is that of Koopman [2] and von Neumann [3]. These works were the foundation of more recent path integral formulations of classical mechanics [4] and the related dequantization procedure of Abrikosov, Gozzi and Mauro [1]. On the other hand, there has been interest in quantization procedures formulated in a quasi-classical language, whereby stochastic terms are added to the equations of classical mechanics. In particular, Nelson [5] and earlier work of Fényes [6] and Weizel [7] showed that the Schrödinger equation can be derived from Newtonian mechanics via the assumption that a classical particle is subjected to Brownian motion with a real diffusion coefficient. Also, Hall and Reginatto 8] have shown that the Schrödinger equation can be derived from the classical equations of motion by adding fluctuations obeying an exact Heisenberg-type equality to the classical momentum. In a similar vein, Reginnato [9] has shown that the Schrödinger equation can be derived by minimization of the Fisher information [10].

In this Letter we present a dequantization procedure whereby classical mechanics is derived from quantum mechanics by suppressing the effects of such "quantum fluctuations". To develop this approach within a consistent mathematical framework, we introduce local deformations of the momentum operator, which correspond to fluctuations of the quantum momentum. These naturally induce an associated deformed kinetic term, which quantifies the amount of "fuzzyness" caused by these fluctuations. Considered as a functional of such deformations, the deformed kinetic term is shown to possess a unique minimum which is seen to be the classical kinetic energy. Furthermore, we show that extremization of the associated deformed action functional introduces an essential nonlinearity to the resulting field equations which are seen to be the classical Hamilton-Jacobi and continuity equations. The minimizing deformation can thus be interpreted as the particular deformation that removes the quantum fluctuations so that the classical case, i.e., dequantization, is attained. Moreover, the minimizing deformation automatically determines an expression for

\footnotetext{
*Electronic address: mosna@ime.unicamp.br

†Electronic address: ihamilto@wlu.ca

‡Electronic address: dellsite@mpip-mainz.mpg.de
} 
the quantum fluctuations which, when added to the classical momentum, leads to the quantum one. This expression is shown to be identical to Nelson's osmotic momentum.

\section{MINIMIZING THE DEFORMED KINETIC TERM}

We begin by considering a local deformation $\boldsymbol{P} \rightarrow \boldsymbol{P}_{\boldsymbol{w}}$ of the quantum momentum operator $\boldsymbol{P}=-i \hbar \boldsymbol{\nabla}$ for an one-particle system (the generalization to many-particle systems of scalar particles is straightforward), with

$$
\boldsymbol{P}_{\boldsymbol{w}} \psi=(\boldsymbol{P}-\boldsymbol{w}) \psi
$$

where $\psi$ is the wavefunction of the system and $\boldsymbol{w}$ is a position-dependent (complex) vector field. ${ }^{1}$ Since our aim is to dequantize the system (thereby leaving the realm of quantum mechanics), there is no a priori reason to assume that $\boldsymbol{P}_{\boldsymbol{w}}$ is Hermitian when $\boldsymbol{w} \neq 0$. Writing $\boldsymbol{w}=\boldsymbol{v}+i \boldsymbol{u}$, where $\boldsymbol{v}$ and $\boldsymbol{u}$ are respectively the real and imaginary parts of $\boldsymbol{w}$, we see that the term $\boldsymbol{v}$ in $\boldsymbol{P}_{\boldsymbol{w}} \psi=-(i \hbar \boldsymbol{\nabla}+\boldsymbol{v}) \psi-i \boldsymbol{u} \psi$ acts in the same way as an electromagnetic field $\boldsymbol{A}$, which is known to change the quantum momentum operator $-i \hbar \boldsymbol{\nabla}$ to $-i \hbar \boldsymbol{\nabla}+\kappa \boldsymbol{A}$, where $\kappa$ is a constant. Therefore, in what follows we restrict the deformations in (1) to those corresponding to imaginary $\boldsymbol{w}$, so that $\boldsymbol{w}=i \boldsymbol{u}$, with $\boldsymbol{u}$ real:

$$
\boldsymbol{P}_{\boldsymbol{u}} \psi=(\boldsymbol{P}-i \boldsymbol{u}) \psi
$$

Let

$$
T=\frac{1}{2 m} \int(\boldsymbol{P} \psi)^{*}(\boldsymbol{P} \psi) d \tau
$$

and

$$
T_{\boldsymbol{u}}=\frac{1}{2 m} \int\left(\boldsymbol{P}_{\boldsymbol{u}} \psi\right)^{*}\left(\boldsymbol{P}_{\boldsymbol{u}} \psi\right) d \tau
$$

be the kinetic terms arising from $\boldsymbol{P}$ and $\boldsymbol{P}_{\boldsymbol{u}}$, respectively, where $m$ is the mass of the particle and $d \tau$ denotes the associated volume element. Integration by parts shows that one can alternatively write

$$
T=\frac{1}{2 m} \int \psi^{*} \boldsymbol{P}^{2} \psi d \tau
$$

as usual, and

$$
T_{\boldsymbol{u}}=\frac{1}{2 m} \int \psi^{*} \boldsymbol{P}_{\boldsymbol{u}}^{\dagger} \boldsymbol{P}_{\boldsymbol{u}} \psi d \tau
$$

where

$$
\boldsymbol{P}_{\boldsymbol{u}}^{\dagger} \psi=(\boldsymbol{P}+i \boldsymbol{u}) \psi
$$

is the adjoint of $\boldsymbol{P}_{\boldsymbol{u}}$. Note that although $\boldsymbol{P}_{\boldsymbol{u}}$ and $\boldsymbol{P}_{\boldsymbol{u}}^{\dagger}$ are, in general, not Hermitian operators, $\boldsymbol{P}_{\boldsymbol{u}}^{\dagger} \boldsymbol{P}_{\boldsymbol{u}}$ is always Hermitian so that $T_{\boldsymbol{u}}$ (like $T$ ) is always a real quantity.

We then have

$$
T_{\boldsymbol{u}}=T+\frac{1}{2 m} \int \rho\left(-\hbar \nabla \cdot \boldsymbol{u}+\|\boldsymbol{u}\|^{2}\right) d \tau,
$$

where $\rho=\psi^{*} \psi$. Note that $T_{\boldsymbol{u}}=T_{\boldsymbol{u}}[\psi, \boldsymbol{u}]$ is a functional of both $\psi$ and $\boldsymbol{u}$. Therefore, the full-fledged variational principle associated with $T_{\boldsymbol{u}}$ should involve minimization with respect to both $\psi$ - and $\boldsymbol{u}$-variations.

A straightforward calculation shows that variation of $T_{\boldsymbol{u}}[\psi, \boldsymbol{u}]$ with respect to $\boldsymbol{u}$ yields

$$
\frac{\delta T_{\boldsymbol{u}}}{\delta \boldsymbol{u}}=\frac{1}{2 m}(2 \rho \boldsymbol{u}+\hbar \nabla \rho) .
$$

\footnotetext{
${ }^{1}$ The most general linear operator acting on $\psi$ can be written, in the position representation, as $\psi(\boldsymbol{r}) \rightarrow \psi^{\prime}(\boldsymbol{r})=\int K\left(\boldsymbol{r}, \boldsymbol{r}^{\prime}\right) \psi\left(\boldsymbol{r}^{\prime}\right) d \boldsymbol{r}^{\prime}$. The deformation considered in (1) is local in the sense that the value of $\left(\boldsymbol{P}_{\boldsymbol{w}}-\boldsymbol{P}\right) \psi$ at a given point $\boldsymbol{r}$ is a function of $\psi(\boldsymbol{r})$ only (in fact, it is given by $-\boldsymbol{w}(\boldsymbol{r}) \psi(\boldsymbol{r}))$.
} 
Therefore, extremization of $T_{\boldsymbol{u}}$ with respect to $\boldsymbol{u}$-variations leads to the critical point

$$
\boldsymbol{u}_{c}=-\frac{\hbar}{2} \frac{\nabla \rho}{\rho} .
$$

This in turn corresponds to the deformed momentum operator

$$
\boldsymbol{P}_{c} \psi=\left(\boldsymbol{P}+\frac{i \hbar}{2} \frac{\boldsymbol{\nabla} \rho}{\rho}\right) \psi,
$$

which has been introduced in [1] from a different perspective (we come back to this point later).

Expanding $T_{\boldsymbol{u}}$ around the critical point yields

$$
T_{\boldsymbol{u}_{c}+\delta \boldsymbol{u}}=T_{\boldsymbol{u}_{c}}+\frac{1}{2 m} \int \rho\|\delta \boldsymbol{u}\|^{2} d \tau,
$$

which shows that the deformation $\boldsymbol{u}_{c}$ of (7) leads to the unique minimum of $T_{\boldsymbol{u}}$, given by

$$
T_{\boldsymbol{u}_{c}}=T-\frac{\hbar^{2}}{8 m} I
$$

where $I$ is the Fisher information [10],

$$
I=\int \frac{(\nabla \rho)^{2}}{\rho} d \tau .
$$

Thus we have shown that there is a unique solution to the deformation parameter $\boldsymbol{u}$ which minimizes the deformed kinetic term $T_{\boldsymbol{u}}$ under $\boldsymbol{u}$-variations. For a pictorial representation of the $\boldsymbol{u}$-dependence of $T_{\boldsymbol{u}}$, see Fig. 1

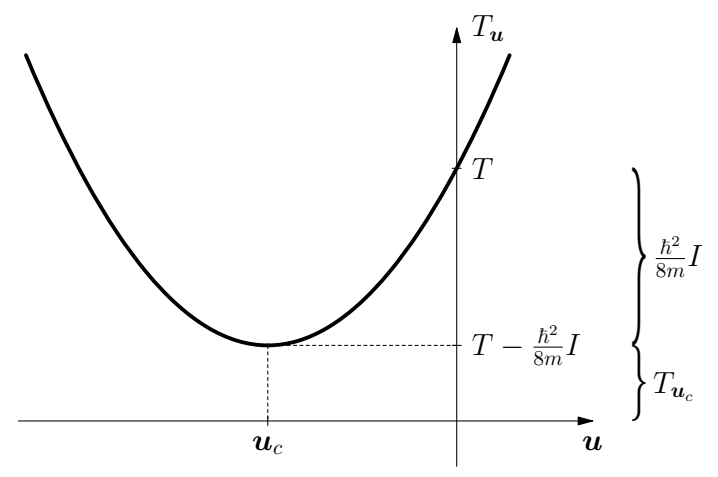

FIG. 1: The minimum of $T_{\boldsymbol{u}}[\psi, \boldsymbol{u}]$ under $\boldsymbol{u}$-variations is attained for $\boldsymbol{u}_{c}=-\frac{\hbar}{2} \frac{\boldsymbol{\nabla} \rho}{\rho}$, with $T_{\boldsymbol{u}_{c}}=T-\frac{\hbar^{2}}{8 m} I$. The plot illustrates this point by pictorially representing the (infinite-dimensional) $\boldsymbol{u}$-space on its abscissa.

A straightforward calculation shows that the action of $\boldsymbol{P}_{c}$ on the wavefunction $\psi=\sqrt{\rho} e^{i S / \hbar}$ of the system is given by

$$
\boldsymbol{P}_{c} \psi=\nabla S \psi
$$

so that, from (4),

$$
T_{\boldsymbol{u}_{c}}=\frac{1}{2 m} \int \rho\|\nabla S\|^{2} d \tau .
$$

This is exactly the mean kinetic energy of a classical ensemble, described by the density $\rho$, with associated Hamilton's principal function $S$ and momentum field $\nabla S[12,13]$. We therefore refer to $T_{\boldsymbol{u}_{c}}$ as the classical kinetic term associated with the ensemble defined by $\rho$ and $S .^{2}$

\footnotetext{
${ }^{2}$ We note that (7) can be rewritten as $\boldsymbol{u}_{c}=\hbar \nabla f$, with $f=-\ln (\rho) / 2$, which is precisely the expression for the deformation function that was introduced in [1] in an ad hoc way. It is now clear that this expression has a deeper justification, since it corresponds to the unique momentum deformation which minimizes the deformed kinetic term.
} 


\section{FROM QUANTUM TO CLASSICAL}

Recall that the Schrödinger equation can be derived from the action functional

$$
\mathcal{S}=\int\left[i \frac{\hbar}{2}\left(\psi^{*} \frac{\partial \psi}{\partial t}-\frac{\partial \psi^{*}}{\partial t} \psi\right)-\frac{1}{2 m}(\boldsymbol{P} \psi)^{*} \cdot(\boldsymbol{P} \psi)-V \psi^{*} \psi\right] d \tau d t
$$

through the usual variational procedure associated with the fields $\psi$ and $\psi^{*}$. The deformation of the momentum operator $\boldsymbol{P} \rightarrow \boldsymbol{P}_{\boldsymbol{u}}$ has the effect of introducing a deformation $\mathcal{S} \rightarrow \mathcal{S}_{\boldsymbol{u}}$, resulting in

$$
\begin{aligned}
\mathcal{S}_{\boldsymbol{u}} & =\int\left[i \frac{\hbar}{2}\left(\psi^{*} \frac{\partial \psi}{\partial t}-\frac{\partial \psi^{*}}{\partial t} \psi\right)-\frac{1}{2 m}\left(\boldsymbol{P}_{\boldsymbol{u}} \psi\right)^{*} \cdot\left(\boldsymbol{P}_{\boldsymbol{u}} \psi\right)-V \psi^{*} \psi\right] d \tau d t \\
& =-\int T_{\boldsymbol{u}}[\psi, \boldsymbol{u}] d t+\int\left[i \frac{\hbar}{2}\left(\psi^{*} \frac{\partial \psi}{\partial t}-\frac{\partial \psi^{*}}{\partial t} \psi\right)-V \psi^{*} \psi\right] d \tau d t
\end{aligned}
$$

which depends on $\boldsymbol{u}$ only through its first term.

This yields a deformed action $\mathcal{S}_{\boldsymbol{u}}[\psi, \boldsymbol{u}]$ whose field equations are obtained by varying both $\psi$ and $\boldsymbol{u}$. From our previous discussion, we see by the form of (15) that extremization with respect to $\boldsymbol{u}$-variations fixes $\boldsymbol{u}$ to be given by (7). Substituting in (15) results in a reduced action, depending only on $\psi$, of the form

$$
\mathcal{S}_{c}[\psi]=\int\left[i \frac{\hbar}{2}\left(\psi^{*} \frac{\partial \psi}{\partial t}-\frac{\partial \psi^{*}}{\partial t} \psi\right)-\frac{1}{2 m}\left(\boldsymbol{P}_{c} \psi\right)^{*} \cdot\left(\boldsymbol{P}_{c} \psi\right)-V \psi^{*} \psi\right] d \tau d t
$$

with $\boldsymbol{P}_{c}$ given by (8). As discussed in [11], (16) is precisely the classical version of the action (14). This can be most easily seen by noting that, when $\psi$ is written in polar form, $\psi=\sqrt{\rho} e^{i S / \hbar}$, (16) becomes

$$
\mathcal{S}_{c}[\rho, S]=-\int \rho\left(\frac{\partial S}{\partial t}+\frac{(\nabla S)^{2}}{2 m}+V\right) d \tau d t
$$

which gives rise, upon variation of $\rho$ and $S$, to the classical Hamilton-Jacobi and continuity equations ${ }^{3}$

$$
\begin{gathered}
\frac{\partial S}{\partial t}+\frac{(\nabla S)^{2}}{2 m}+V=0 \\
\frac{\partial \rho}{\partial t}+\nabla \cdot\left(\rho \frac{\nabla S}{m}\right)=0
\end{gathered}
$$

Thus we have shown that the procedure of "deforming the momentum and extremizing its associated deformed action functional" can be effectively regarded as a dequantization method, at least for scalar particles. Note that the choice of deformed momentum that extremizes its associated deformed action is given by $\boldsymbol{P}_{c}$ of (8). This can be thought of as a classical version of the momentum operator since its associated kinetic term, $T_{\boldsymbol{u}_{c}}$, is the mean kinetic energy of the classical ensemble defined by $\rho$ and $S$ (as discussed earlier), and its associated deformed action, $\mathcal{S}_{c}$, yields the classical Hamilton-Jacobi and continuity equations. Further justification on the interpretation of $\boldsymbol{P}_{c}$ as a classical version of the momentum operator can be found in 11], where $\boldsymbol{P}_{c}$ was introduced from a different perspective and shown to be equivalent to an expression introduced by Hall in [14] that gives the best classical estimate of the momentum which is compatible with simultaneous knowledge of the position of the system.

In the quantization procedure of Nelson [5] a classical particle is subjected to Brownian motion. In addition to its classical velocity, a Brownian particle has a velocity due to the osmotic force, that Nelson terms the osmotic velocity (which is half the difference between the forward and backward drift velocities). From Einstein's theory, the osmotic velocity is given by $\nu \nabla \rho / \rho$ where $\nu$ is the diffusion coefficient. Since macroscopic bodies do not appear to be subjected to Brownian motion, Nelson assumes that $\nu$ is inversely proportional to the particle mass and makes the ansatz $\nu=\hbar / 2 m$. Then the corresponding osmotic momentum, which is the term added to the classical momentum

\footnotetext{
${ }^{3}$ Alternatively, we could have directly calculated the Euler-Lagrange equations associated with the deformed action $\mathcal{S}_{\boldsymbol{u}}[\psi, \boldsymbol{u}] . \quad$ The Euler-Lagrange equations relative to $\boldsymbol{u}$ then yield $2 \rho \boldsymbol{u}+\hbar \nabla \rho=0$, while the Euler-Lagrange equations relative to $\psi$ and $\psi^{*}$ yield $i \hbar \frac{\partial \psi}{\partial t}=-\frac{\hbar^{2}}{2 m} \nabla^{2} \psi+\left[V+\frac{1}{2 m}\left(\boldsymbol{u}^{2}-\hbar \nabla \cdot \boldsymbol{u}\right)\right] \psi=0$. Isolating $\boldsymbol{u}$ from the former equation and substituting into the latter then yields, in terms of $\rho$ and $S$, [18] and [19].
} 
to give the quantum one, is $(\hbar / 2) \nabla \rho / \rho$. This expression is seen to be identical to minus our $\boldsymbol{u}_{c}$ of (7). This is no coincidence and can be qualitatively understood as follows. In Nelson's quantization approach, quantum fluctuations (expressed as the osmotic momentum) are explicitly added to $\boldsymbol{P}_{c}$, thereby resulting in the quantum momentum. In our dequantization approach, the quantum fluctuations latent in $\boldsymbol{P}$ are stripped off by the process of minimizing $T_{\boldsymbol{u}}$, thereby isolating the classical momentum. In the process, our dequantization approach automatically identifies the expression for $-\boldsymbol{u}_{c}$ (cf (7)).

\section{DISCUSSION}

We have presented a dequantization procedure based on a variational principle whereby quantum fluctuations latent in the quantum momentum are suppressed. To this end, we added generic local deformations $\boldsymbol{u}$ to the quantum momentum operator $\boldsymbol{P}$. Such deformations are independent of $\psi$ and consequently the deformed momentum operator $\boldsymbol{P}_{\boldsymbol{u}}$ is linear in $\psi(\operatorname{cf}(2))$. However, after extremization of the associated deformed kinetic term $T_{\boldsymbol{u}}, \boldsymbol{u}$ becomes dependent on $\psi$ (and fixed to $\boldsymbol{u}=-\frac{\hbar}{2} \frac{\boldsymbol{\nabla} \rho}{\rho}$ ), giving rise to the nonlinear classical momentum operator $\boldsymbol{P}_{c}$ of (8). Furthermore, extremization of the associated deformed action, $\mathcal{S}_{\boldsymbol{u}}$, gives rise to the classical Hamilton-Jacobi and continuity equations (18) and (19), so that dequantization is attained.

For a classical system described by a probability density there is uncertainty in the position (and momentum). For the corresponding quantum system there is additional uncertainty. The dequantization method presented here removes the additional part of the uncertainty that is quantum leaving only the uncertainty that is classical and it does this in a "minimalist" way — without introducing any artifacts - through a deformation procedure based on a variational principle. As a result of the dequantization procedure the quantum momentum fluctuations are suppressed and, in this sense, the momentum-space localization of the system (thereupon considered as a classical ensemble) is increased. However, the spatial localization of the system is unchanged as this quantity is determined by $\rho$ which is unaffected by the dequantization process (in fact, as noted above, $\rho$ determines the deformation function). For a system which is more spatially localized both the quantum kinetic term and the Fisher information are larger and in the limit of extreme spatial localization both become infinite but the classical kinetic term can remain finite.

The approach presented here may shed light on the quantum-classical transition, since the passage from the linear equations of quantum mechanics to the nonlinear equations of classical mechanics is made salient through the deformation function $\boldsymbol{u}$. A remarkable effect of this linearity $v s$ nonlinearity issue is the fundamentally different characterization of the concept of chaos in quantum and classical systems [15]. Consideration of the deformed action $\mathcal{S}_{\boldsymbol{u}}$ for generic fixed values of $\boldsymbol{u}$, different from $\mathbf{0}$ and $\boldsymbol{u}_{c}$, may contribute novel insights in this regard. It may also facilitate novel formulations of semiclassical mechanics.

As noted previously, dequantization is "a set of rules which turn quantum mechanics into classical mechanics" [1]. For the dequantization procedure proposed here these rules are "deform the momentum and extremize its associated deformed action functional". It would be interesting to investigate how this procedure (developed here for scalar particles) can be extended to other contexts, such as particles with internal degrees of freedom.

\section{Acknowledgments}

We thank B. Dünweg, E. Guéron, M. J. W. Hall and M. Reginatto for helpful comments. RAM acknowledges FAPESP for financial support. IPH acknowledges funding from NSERC and thanks Wilfrid Laurier University and the Fields Institute for support.

[1] A. A. Abrikosov Jr, E. Gozzi and D. Mauro, Ann. Phys. 317, 24 (2005).

[2] B. O. Koopman, Proc. Nat. Acad. Sci. USA 17, 315 (1931).

[3] J. von Neumann, Ann. Math. 33, 587 (1932).

[4] E. Gozzi, M. Reuter and W.D. Thacker, Phys. Rev. D 40, 3363 (1989); 46, 757 (1992).

[5] E. Nelson, Phys. Rev. 150, 1079 (1966); E. Nelson, Dynamical Theories of Brownian Motion (Princeton Univ. Press, Princeton, 1967).

[6] I. Fényes, Z. Physik 132, 81 (1952).

[7] W. Weizel, Z. Physik 134, 264 (1953); 135, 270 1953; 136, 582 (1954).

[8] M. J. W. Hall and M. Reginatto, J. Phys. A 35, 3289 (2002), quant-ph/0102069

[9] M. Reginatto, Phys. Rev. A 58, 1775 (1998).

[10] R. A. Fisher, Proc. Cambridge Philos. Soc. 22, 700 (1925). 
[11] R. A. Mosna, I. P. Hamilton and L. Delle Site, J. Phys. A 38, 3869 (2005), quant-ph/0504124

[12] H. Goldstein, Classical Mechanics, 2nd ed. (Addison-Wesley, Reading, MA, 1980).

[13] P. R. Holland, The Quantum Theory of Motion (Cambridge University Press, Cambridge, 1993).

[14] M. J. W. Hall, Phys. Rev. A 62, 12107 (2000), quant-ph/9912055 M. J. W. Hall, Phys. Rev. A 64, 52103 (2001), quant-ph/0107149

[15] See, e.g., M. C. Gutzwiller, Chaos in Classical and Quantum Mechanics (Springer-Verlag, New York, 1990). 